\title{
Density-Induced Breaking of Pairs in the Attractive Hubbard Model
}

\author{
Bumsoo Kyung, ${ }^{1}$ E. G. Klepfish, ${ }^{2}$ and P. E. Kornilovitch ${ }^{1}$ \\ ${ }^{1}$ Max-Planck-Institut für Physik komplexer Systeme, Nöthnitzer str. 38, D-01187, Dresden, Germany \\ ${ }^{2}$ Department of Physics, King's College London, Strand, London, WC2R 2LS, UK
}

(5 September 1997)

\begin{abstract}
A conserving $T$-matrix approximation is applied to the two-dimensional attractive Hubbard model in the low-density regime. A set of self-consistent equations is solved in the real-frequency domain to avoid the analytic continuation procedure. By tuning the chemical potential the particle density was varied in the limits $0.01<n<0.18$. For the value of the attractive potential $U=8 t$ the binding energy of pairs monotonically decreases with increasing $n$, from its zero-density limit $2.3 t$ and vanishes at a critical density $n_{c r}=0.19$. A pairing-induced pseudogap in the single-particle density of states is found at low densities and temperatures.
\end{abstract}

PACS numbers: 71.10.Fd, 74.20.Mn

The pseudogap state of underdoped cuprates is currently one of the central topics in the studies of hightemperature superconductivity (HTSC). The pseudogap (PG) is believed to be responsible for marked deviations from Fermi-liquid behavior observed in a variety of probes. Indications from NMR [1,2], specific heat measurements [3], transport [4,5], optics [6,7] were followed by more recent angle-resolved photoemission [8], Raman [9] and core-electron photoemission [10] studies. A consensus seems to be emerging that the PG is of crucial importance for understanding HTSC because it is intimately related to superconductivity. The PG has the same symmetry as the superconducting gap [8,9,11], it decreases with hole doping [3,5, 12] and has a universal doping dependence when measured in units of the maximum critical temperature 11.

The PG state gets a simple explanation in phenomenological models which assume an effective short-range attractive interaction between holes doped into a MottHubbard insulator 13, 14. PG is then associated with the binding energy $\Delta$ of hole pairs. Superconductivity sets in at lower temperatures $T_{c} \sim n_{\text {pairs }} / m_{\text {pairs }}$ [15]. Thus, at small hole densities $\Delta$ and $T_{c}$ are well separated. Microscopic origin of such an attraction may be different varying from pure electronic [16] to pure phononic [13] mechanisms, see also [17.

In this Letter we address the doping dependence of the PG. It has already been discussed by Alexandrov, Kabanov, and Mott in 18]. They found that the binding energy of pairs decreases with doping as a result of the screening of the long-range electron-phonon interaction and renormalization of the inter-hole coupling. Here we will show that PG decreases with doping even in the case of short-range inter-hole interaction and without coupling renormalization. This is a pure many-body effect which is expected on the following grounds. If the coupling exceeds the binding threshold then, at low density, the carriers form non-overlapping pairs. As the density increases pairs begin to overlap and fermions, which constitute pairs, begin to form a Fermi sea. This process increases the energy of the system per particle hence raising the energy of the two-particle level. Under certain conditions this level could be pushed up to the single-particle continuum. Near the critical density the binding energy and the life-time of pairs become small and pairs break up.

In order to verify this idea we study the twodimensional attractive Hubbard model which is the simplest fermion lattice model with attraction. The model is defined by Hamiltonian

$$
H=\sum_{\mathbf{k} \sigma}\left(\varepsilon_{\mathbf{k}}-\mu\right) c_{\mathbf{k} \sigma}^{\dagger} c_{\mathbf{k} \sigma}-\frac{|U|}{N} \sum_{\mathbf{k p q}} c_{\mathbf{k} \uparrow}^{\dagger} c_{\mathbf{k}+\mathbf{q} \uparrow} c_{\mathbf{p} \downarrow}^{\dagger} c_{\mathbf{p}-\mathbf{q} \downarrow}
$$

written in the standard notation. $\varepsilon_{\mathbf{k}}=-2 t\left(\cos k_{x}+\right.$ $\left.\cos k_{y}\right)$ is the single-particle bare spectrum, $|U|$ is the coupling strength, $N$ is the total number of sites in the system and chemical potential $\mu$ determines the average particle density. We will be interested in the low-density regime of the model. In this regime one can make use of the small gas parameter and select only ladder diagrams in the diagrammatic representation of the Bethe-Salpeter equation [19,20]. This procedure leads to the $T$-matrix approximation for the Hubbard model [21] which we formulate here in the real-frequency domain. The singleparticle spectral function $A(\mathbf{k}, \omega)$ is determined by the real and imaginary parts of the self-energy $\Sigma^{\prime}$ and $\Sigma^{\prime \prime}$

$$
A(\mathbf{k}, \omega)=\frac{-2 \Sigma^{\prime \prime}(\mathbf{k}, \omega)}{\left[\omega-\left(\varepsilon_{\mathbf{k}}-\mu\right)-\Sigma^{\prime}(\mathbf{k}, \omega)\right]^{2}+\left[\Sigma^{\prime \prime}(\mathbf{k}, \omega)\right]^{2}} .
$$

The self-energy is expressed, in turn, via the real and imaginary parts $T^{\prime}$ and $T^{\prime \prime}$ of the $T$-matrix

$$
\Sigma^{\prime}(\mathbf{k}, \omega)=\frac{1}{N} \sum_{\mathbf{q}} \int \frac{d \omega_{1}}{2 \pi} A\left(\mathbf{q}-\mathbf{k}, \omega_{1}\right) \times
$$




$$
\begin{aligned}
& \times\left[f_{F}\left(\omega_{1}\right) T^{\prime}\left(\mathbf{q}, \omega+\omega_{1}\right)+\int \frac{d \omega_{2}}{\pi} \frac{f_{B}\left(\omega_{2}\right) T^{\prime \prime}(\mathbf{q}}{\omega_{2}-\omega_{1}}\right. \\
& \Sigma^{\prime \prime}(\mathbf{k}, \omega)=\frac{1}{N} \sum_{\mathbf{q}} \int \frac{d \omega_{1}}{2 \pi} A\left(\mathbf{q}-\mathbf{k}, \omega_{1}\right) \times \\
& \times T^{\prime \prime}\left(\mathbf{q}, \omega+\omega_{1}\right)\left[f_{F}\left(\omega_{1}\right)+f_{B}\left(\omega+\omega_{1}\right)\right]
\end{aligned}
$$

where $f_{F, B}(\omega)=[\exp (\beta \omega) \pm 1]^{-1}$ are Fermi- and Bosefunctions respectively, and $\beta=1 / T$ is the inverse absolute temperature. Finally, the $T$-matrix is expressed via the spectral function as follows

$$
\begin{gathered}
T(\mathbf{q}, \omega)=\frac{-|U|}{1-|U| \int \frac{d \omega_{1}}{2 \pi} \frac{B\left(\mathbf{q}, \omega_{1}\right)}{\omega-\omega_{1}}+i \frac{|U|}{2} B(\mathbf{q}, \omega)} \\
B(\mathbf{q}, \omega)=\frac{-1}{N} \sum_{\mathbf{k}^{\prime}} \int \frac{d \omega_{1}}{2 \pi} A\left(\mathbf{k}^{\prime}, \omega_{1}\right) A\left(\mathbf{q}-\mathbf{k}^{\prime}, \omega-\omega_{1}\right) \tanh \frac{\beta \omega_{1}}{2} .
\end{gathered}
$$

The integrals with singular kernels in Eqs.(3) and (5) are understood in the principal value sense. The set of equations (2)-(6) is to be solved self-consistently for given $|U|, \mu$ and temperature $T$, the particle density given by:

$$
\frac{n}{2}=\frac{1}{N} \sum_{\mathbf{k}} \int_{-\infty}^{\infty} \frac{d \omega}{2 \pi} A(\mathbf{k}, \omega) f_{F}(\omega)
$$

Single-particle energies are associated with sharp peaks in the spectral function $A(\mathbf{k}, \omega)$. Analogously, the energies of two-particle bound states can be inferred from peaks of the imaginary part of the $T$-matrix $T^{\prime \prime}(\mathbf{q}, \omega)$ which is proportional to the spectral function of the two-particle propagator with total quasi-momentum q, $A_{2}(\mathbf{q}, \omega)=-T^{\prime \prime}(\mathbf{q}, \omega) /|U|$. The difference between the pair energy and the doubled single-particle energies, particularly the dependence of this difference on the density of particles, is the main object of this work.

The method of numerical solution of the set of equations is well-known: one has to iterate Eqs. (2)-(6) starting with some initial guess for the spectral function and to use the Fast Fourier Transform (FFT) algorithm to speed up the calculation of the integrals. We note that in previous analogous studies 222 24] the equations were formulated in the imaginary-time domain which required an analytic continuation of the iteration results to real frequencies. This ill-posed problem of numerical analytic continuation leads to ambiguities in the details of reconstructed functions. Therefore we prefer to deal directly with the real-frequency equations. We have observed that the singular structure of the kernels in Eqs. (2)(6) is not an obstacle for the FFT-integration and does not slow down the convergence of the iterative process.

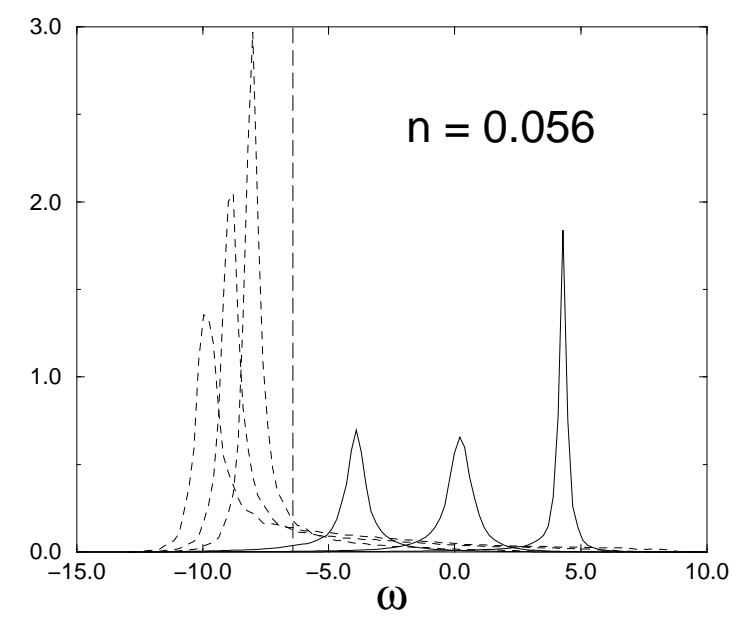

FIG. 1. Spectral function $A(\mathbf{k}, \omega) /(2 \pi)$ (solid lines) and $A_{2}(\mathbf{k}, \omega)=-T^{\prime \prime}(\mathbf{k}, \omega) /|U|$ (dashed lines) for three values of lattice momentum, $\mathbf{k}=(0,0),(\pi / 2, \pi / 2)$ and $(\pi, \pi)$ (from left to right). Energy is measured with respect to the on-site single-particle level i.e. $A(\mathbf{k}, \omega)$ are shifted by $\mu$ and $A_{2}(\mathbf{k}, \omega)$ by $2 \mu$ from results of self-consistent solution. The position of the chemical potential $\mu=-6.4 t$ is shown by the vertical long-dashed line. $|U|=8 t$, temperature $T=1.0 t$ and the corresponding particle density is $n=0.056$.

Our calculations were done on a $64 \times 64$-site lattice and on a uniform mesh of 512 points in the frequency interval $-20 t<\omega<30 t$. Eqs. (2)-(6) were iterated until the relative difference between two successive iterations fell below 0.01 (usually 20-25 iterations). Note, that no artificial damping factor was used in the iteration process. The resulting spectral functions were checked against the normalization property $\int \frac{d \omega}{2 \pi} A(\mathbf{k}, \omega)=1$ (for other sum rules for the Hubbard model see e.g. 25.23]) which were found to be satisfied within $5 \%$ accuracy for each $\mathbf{k}$ (normalization was accurate within 1\%). The temperature was taken $T=1.0 t$.

Figs. 1 and 2 show results of iterations for two different values of the chemical potential $\mu=-6.4 t$ and $\mu=$ $-5.8 t$ corresponding to particle densities $n=0.056$ and $n=0.111$ respectively. To obtain the binding energy of a bound state with total quasi-momentum q one has to compare the position of the corresponding peak in $A_{2}(\mathbf{q}, \omega)$ with the doubled position of a peak in spectral function $A(\mathbf{k}, \omega)$ taken at half momentum $(\mathbf{q} / 2)$. Thus, in Fig. 1 the binding energy of the pair with $\mathbf{q}=(0,0)$ is $2 E_{1}(0,0)-E_{2}(0,0) \approx 2.3 t$, the one of the pair with $\mathbf{q}=$ $(\pi, \pi)$ is $2 E_{1}(\pi / 2, \pi / 2)-E_{2}(\pi, \pi) \approx 8.0 t$. An analysis of Figs. 1 and 2 and analogous data for seven other densities in the region $0.01 \leq n \leq 0.18$ (not shown) reveals the following common trends. i) The single-particle peak as a function of momentum $\mathbf{k}$ spans an energy interval of width $\approx 8 t$ for all the densities studied. This implies 


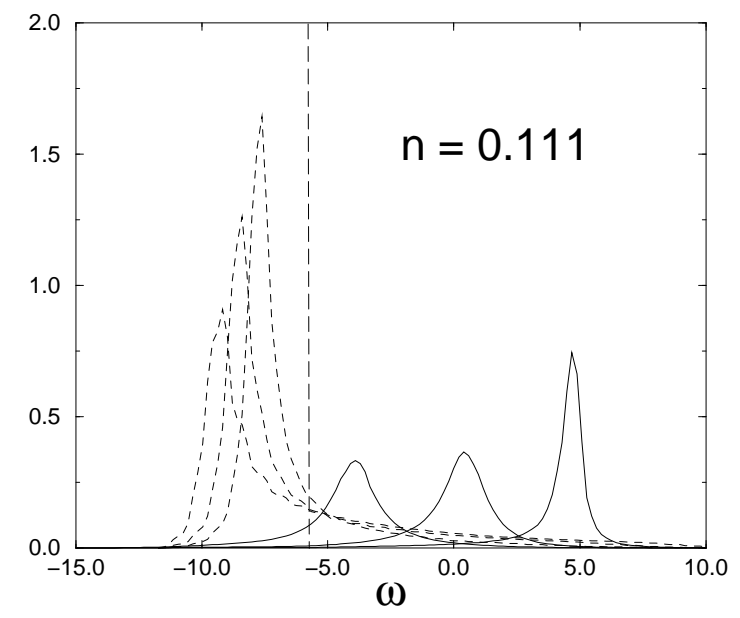

FIG. 2. The same as Fig. 1 but for chemical potential $\mu=-5.8 t$ corresponding to a larger density $n=0.111$.

small effect of the interaction on the shape and width of the single-particle energy spectrum. The same conclusion has been reached in Ref. [23]. ii) The lower boundary of the single-particle band $E_{1}(0,0)$ shifts towards negative values as density increases. However, the shift observed is smaller than the mean-field value $-|U| n / 2$. Apparently, this is due to the correlation effects. At $|U|=8 t$ the effective radius of a pair is $\approx 1-2$ lattice spacings, see e.g. [26]. Therefore the interaction of a particle with spin $\sigma$ excited to the continuum spectrum, with a bound particle of spin $-\sigma$ is effectively screened by the latter's spin $\sigma$ partner. iii) The energy of the two-particle state increases with the density, as indeed was expected on the grounds of the qualitative considerations. iv) The single particle and the pair spectral functions become narrower at large lattice momenta. The life-time of the bound states with large momenta increases which results from reduced phase space of the scattering process into two single-particle states. v) The binding energy of the pair with the highest momentum $\mathbf{q}=(\pi, \pi)$ is always close to $|U|$ (which is equal to $8 t$ in our case). This result of a fully self-consistent treatment is in perfect agreement with the exact solution of the two-body problem (the zero-density limit) [27] and the general theory of the $\eta$ resonance 28 according to which a collective mode at $\mathbf{q}=(\pi, \pi)$ with binding energy $|U|$ always exists in the attractive Hubbard model.

At low density only states with small momenta are occupied. Therefore we now concentrate on the properties of pairs with $\mathbf{q}=(0,0)$. We have analysed the doping-dependence of the binding energy using solutions of the self-consistent equations for nine different densities in the interval $0.01 \leq n \leq 0.18$. For the smallest density studied, $n=0.01$, the binding energy was found to be $\approx 2.3 t$. For the two-particle problem the binding

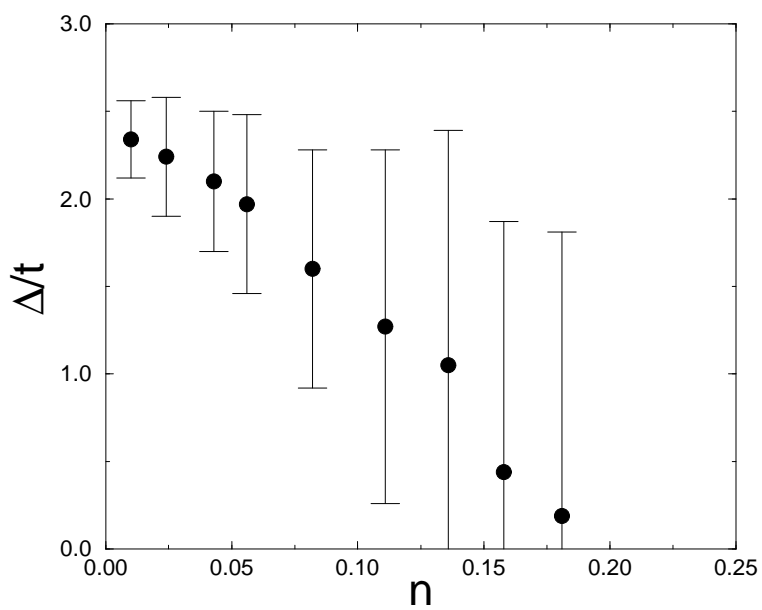

FIG. 3. Pair binding energy vs. particle density. The points are determined from the position of the maxima of peaks of spectral functions. Bars indicate the width of peaks.

energy in the ground state is $\Delta(n=0)=2.1 t$ [27]. The proximity of these two values confirms the correctness of our numerical treatment of the self-consistent equations. The small difference is apparently due to the finite-size effects. The density dependence of the binding energy is shown in Fig. 3. It is a monotonically decreasing function which vanishes at $n_{c r} \approx 0.19$. The points have been calculated from the positions of the maxima of one- and two-particle spectral functions. The peaks' widths are associated with finite life-time of the excitations. The bars in Fig.3 indicate the width (the inverse life-time) of the two-particle spectral weight. Obviously, if the energy gap between the bound states and the single-particle continuum diminishes the scattering process of the pair into two single-particle states intensifies. This should lead to decrease of the life-time and widening of the peaks. This is exactly what is seen in the self-consistent results. We have checked that the decrease of the binding energy is to be attributed mainly to the increase of the energy of two-particle states. There is also a contribution from the negative shift of single-particle continuum, but we have found this process to account for $\approx 10 \%$ of the whole effect. It is also clear that the region of stability of the bound states depends on the coupling constant: it expands for larger $|U|$ 's and shrinks for smaller ones. We have repeated all the calculations for $|U|=4.0 t$ and $T=0.2 t$ and found that for density $n=0.10$ the pair with $\mathbf{q}=(0,0)$ is not a well-defined excitation, its binding energy and life-time being too small. The pair excitation separates from the single-particle continuum at $\mathbf{q} \approx(\pi / 2, \pi / 2)$. At the corner of the Brillouin zone the pair's binding energy is $\approx|U|=4.0 t$. Therefore, the coupling is to be increased if one requires well-defined pair excitations at $\mathbf{q}=(0,0)$. At large couplings the conver- 


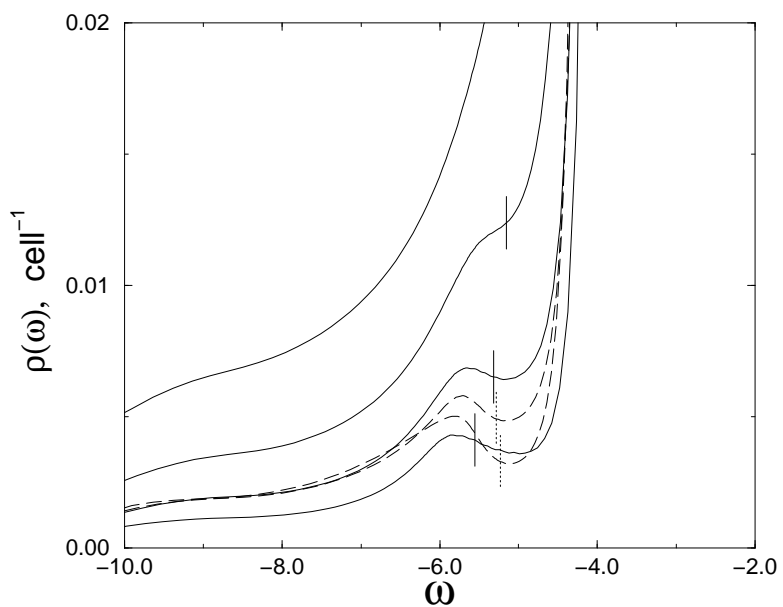

FIG. 4. The low-energy part of the density of states for $|U|=8 t$. Solid lines are for $T=0.5 t$ and densities $n=1.0,1.7,3.3$ and $6.3 \%$ (from the lowest curve upwards). Dashed lines are $n=1.7 \%$ and $T=0.4 t$ (upper curve) and $T=0.3 t$. Vertical lines mark the energies $\omega_{0}$ such that $\int_{-\infty}^{\omega_{0}} d \omega \rho(\omega)=n$.

gence of the iterative process worsens but restores with increasing temperature. Thus, we were able to trace the destruction of pairs in full at $|U|=8.0 t$ and $T=1.0 t$.

The density dependence of the pair binding energy found in our calculations is similar to that of the pseudogap observed in the underdoped regime of high- $T_{c}$ superconductors. This supports the real-space pairing as the cause for the pseudogap phenomenon. In order to test and clarify this relation further we calculated the singleparticle density of states $\rho(\omega)$ from the solution of the $T$ matrix equations. The low-energy part of $\rho(\omega)$ is shown in Fig. 4. At small $n$ we observe an extra peak in $\rho(\omega)$ which is apparently due to the presence of bound states, since its separation from the main part of $\rho(\omega)$ is approximately the binding energy. (Compare with the atomic limit of the Hubbard model, see e.g. [23].) At larger $n$ the feature shifts to higher energies and finally disappears thereby qualitatively repeating the $n$-dependence of the binding energy discussed above. Two comments are to be added. First, the density of states is an integral of the single-particle spectral function over momenta $\mathbf{k}$. Since at different $\mathbf{k}$ the additional maxima appear at different energies the feature is less pronounced in $\rho(\omega)$ than in individual $A(\mathbf{k}, \omega)$. Second, upon lowering the temperature the local minimum in $\rho(\omega)$ deepens, see the dashed curves in Fig. 6 . This implies the opening of a pseudogap at low temperatures, as experimentally observed in the underdoped cuprates. Note that the position of the peak is not affected by temperature which confirms that it is the pair binding energy.

In conclusion, we have studied the low-density regime of the two-dimensional attractive Hubbard model by means of the self-consistent $T$-matrix approximation. The set of self-consistent equations was solved in the realfrequency domain to avoid uncertainties related to the analytic continuation procedure. The binding energy of pairs was found to be a monotonically decreasing function of the total density. We interprete this as a pure many-body effect resulting from the rise of two-particle levels to the single-particle continuum due to the packing effect. We believe this effect is generic to low-density regimes of fermionic models with attraction and it does not depend on the dimensionality or particular details of the interaction. In particular it should remain in more realistic models of low-density hole systems such as the nearest-neighbors attraction model with $d$-symmetrical bound states. We observe also a pairing-induced pseudogap in the density of states with the characteristic size equal to the binding energy of pairs. The pseudogap develops at low densities upon lowering temperature.

We acknowledge valuable discussions on the subject with A.S. Alexandrov, A. M. Dyugaev, S. Flach, P. Fulde, A. O. Gogolin, R. B. Laughlin, A. A. Nersesyan, Yu. N. Ovchinnikov, and E. R. Pike. EGK has been supported by EPSRC grant GR/J18675.

[1] M. Takigawa et al., Phys. Rev. B 43, 247 (1991).

[2] G. V. M. Williams et al., Phys. Rev. B 51, 16503 (1995).

[3] J. W. Loram et al., Phys. Rev. Lett. 71, 1740 (1993); Physica (Amsterdam) 235C-240C, 134 (1994).

[4] T. Ito, K. Takenaka, and S. Uchida, Phys. Rev. Lett. 70, 3995 (1993).

[5] B. Batlogg et al., Physica (Amsterdam) 235C-240C, 130 (1994).

[6] L. D. Rotter et al., Phys. Rev. Lett. 67, 2741 (1991).

[7] C. C. Homes et al., Phys. Rev. Lett. 71, 1645 (1993).

[8] A. G. Loeser et al., Science, 273, 325 (1996).

[9] R. Nemetschek et al., Phys. Rev. Lett. 78, 4837 (1997).

[10] O. Tjernberg et al., Phys. Rev. Lett. 79, 499 (1997).

[11] G. V. M. Williams et al., Phys. Rev. Lett. 78, 721 (1997).

[12] A. V. Puchkov et al., Phys. Rev. Lett. 77, 3212 (1996).

[13] A.S. Alexandrov and N. F. Mott, Superc. Sci. Tech. 6, 215 (1993); High-Temperature Superconductors and other Bose Liquids (Taylor \& Francis, 1994).

[14] M. Randeria et al., Phys. Rev. Lett. 69, 2001 (1992); N. Trivedi and M. Randeria, Phys. Rev. Lett. 75, 312 (1995).

[15] Y. J. Uemura

et al., Nature 364, 605 (1993); Y. J. Uemura, preprint cond-mat/9706151.

[16] E. Dagotto, Rev. Mod. Phys. 66, 763 (1994).

[17] R. Micnas, J.Ranninger, and S. Robaszkiewicz, Rev. Mod. Phys. 62, 113 (1990).

[18] A.S. Alexandrov, V. V.Kabanov, and N.F. Mott, 
Phys. Rev. Lett. 77, 4796 (1996).

[19] V. M. Galitskii, Sov. Phys. JETP 34, 104 (1958).

[20] L. P. Kadanoff and G. Baym, Quantum Statistical Mechanics (Benjamin, 1962).

[21] J. Kanamori, Prog. Theor. Phys. 30, 275 (1963).

[22] R. Frésard, B. Glasser, and P. Wölfle, J. Phys. Cond. Matt. 4, 8565 (1992).

[23] R. Micnas et al., Phys. Rev. B 52, 16223 (1995).

[24] J. R. Engelbrecht et al., preprint cond-mat/9705166.

[25] W. Nolting, Z. Phys. 255, 25 (1972).

[26] P. E. Kornilovitch, in Polarons and Bipolarons in High$T_{c}$ Superconductors and Related Materials, ed. by E. K.H. Salje et al., (Cambridge University Press, 1995), p.367.

[27] The equation for the pair spectrum in the two-body problem is $1=-|U| \sum_{\mathbf{k}}\left[E_{2}(\mathbf{q})-\varepsilon_{\mathbf{k}}-\varepsilon_{\mathbf{q}-\mathbf{k}}\right]^{-1}$. For $\mathbf{q}=(\pi, \pi)$ one obtains $E_{2}=-|U|$.

[28] E. Demler et al., Int. J. Mod. Phys. B 10, 2137 (1996). 